\title{
SORÇÃO E PERSISTÊNCIA DA SORGOLEONA EM UM LATOSSOLO VERMELHO-AMARELO
}

Antonio J. Demuner*, Luiz C. A. Barbosa, Luiz S. Chinelatto Jr. e César Reis

Departamento de Química, Universidade Federal de Viçosa, 36571-000 Viçosa - MG

Antonio A. Silva

Departamento de Fitotecnia, Universidade Federal de Viçosa, 36571-000 Viçosa - MG

Recebido em 28/4/04; aceito em 20/10/04; publicado na web em 2/2/05

\begin{abstract}
SORPTION AND PERSISTENCE OF SORGOLEONE IN RED-YELLOW LATOSOL. The root exudates produced by sorghum contain a biologically active constituent known as sorgoleone. The behavior of sorgoleone in a Red-Yellow Latosol was studied. The sorption model of sorgoleone in soil was better adjusted to the Freundlich equation, through the coefficients $\mathrm{K}_{\mathrm{f}}$ (capacity of sorption) and $1 / \mathrm{n}$ (linearity of the isotherm). The persistence of sorgoleone in soil and its possible degradation were also evaluated by monitoring their residues in the soil along the time. Recovery rate of sorgoleone from the soil reached $93 \%$ after $24 \mathrm{~h}$. It was verified that sorgoleone is strongly sorbed in the soil and its half-life is 10 days, under the experimental conditions. The presence of sorgoleone or its metabolites was not detected in the soil after 60 days.
\end{abstract}

Keywords: allelopathy; sorghum; sorption isotherm.

\section{INTRODUÇÃO}

O fenômeno da alelopatia engloba todos os tipos de interações planta-planta, planta-microrganismos e microrganismos-microrganismos mediadas por compostos químicos. Os compostos relacionados a esse fenômeno são genericamente denominados aleloquímicos ${ }^{1}$.

A alelopatia está fortemente associada com a competição existente entre os organismos por recursos naturais do meio, tais como, água, luz e nutrientes. Os aleloquímicos produzidos por uma planta, por exemplo, podem influenciar a vegetação de um local, a sucessão de plantas, a indução de dormência e a preservação de sementes, a germinação de sementes e de esporos de fungos, a produtividade de culturas etc ${ }^{2}$.

Dentre as diversas espécies de vegetais cultivadas pelo homem, o sorgo é uma das que apresenta acentuada atividade alelopática, conhecida e estudada desde o início do século $\mathrm{XX}^{3}$. Estudos de campo realizados no estado do Nebraska (USA) mostraram que a palhada do sorgo, incorporada ao solo, é efetiva no controle de plantas daninhas ${ }^{4}$. No Brasil, a maioria do sorgo é utilizado, principalmente, como grãos e forrageira para alimentação animal, ou como fonte de palhada para o plantio direto, principalmente de soja e milho na região do cerrado.

Dentre as diversas substâncias responsáveis pela atividade alelopática de culturas de sorgo encontram-se glicosídios cianogênicos, taninos, flavonóides, ácido ferúlico, ácido sirígico, ácido vanílico etc ${ }^{5-8}$. Entretanto, estudos recentes têm demonstrado que o principal composto responsável pela alelopatia de culturas de sorgo é a quinona, denominada sorgoleona (Figura 1) ${ }^{9,10}$. Este composto apresentou efeito inibitório do crescimento tanto de raízes como da parte aérea de espécies cultivadas e daninhas ${ }^{4,9-11}$.

Apesar da existência de diversos estudos relatando a eficácia da sorgoleona no controle de plantas daninhas ${ }^{11,12}$, sobre seu modo de ação ${ }^{13-15}$ e seu uso como modelo para o preparo de novos

*e-mail: ademuner@ufv.br

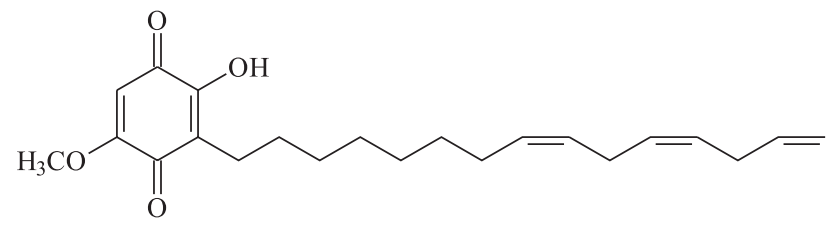

Figura 1. Fórmula estrutural da sorgoleona

herbicidas ${ }^{16,17}$, nenhum trabalho sobre a sorção e a persistência desse composto em solo brasileiro foi ainda publicado.

A sorção de um composto químico no solo pode ser avaliada por meio de isotermas de adsorção, que são funções que relacionam a quantidade adsorvida com a concentração de equilíbrio na solução. Existem vários modelos que podem ser empregados para descrever a sorção de herbicidas em solos, todavia, o modelo de Freundlich é o mais utilizado ${ }^{18-25}$. Esse modelo possui bases empíricas, sendo representado pela equação $\mathrm{X} / \mathrm{m}=\mathrm{K}_{\mathrm{f}} \mathrm{C}_{\mathrm{e}}{ }^{1 / n}$ ou $\log \mathrm{X} /$ $\mathrm{m}=\log \mathrm{K}_{\mathrm{f}}+(1 / \mathrm{n}) \log \mathrm{C}_{\mathrm{e}}^{25}$. Nesse caso $\mathrm{X} / \mathrm{m}$ é a massa de herbicida sorvida por unidade de massa de solo $\left(\mu \mathrm{g} \mathrm{g}^{-1}\right) ; \mathrm{K}_{\mathrm{f}}$, a constante de Freundlich; $\mathrm{C}_{\mathrm{e}}$, concentração do herbicida em equilíbrio com o solo $\left(\mu \mathrm{g} \mathrm{mL}^{-1}\right)$ e $1 / \mathrm{n}$, o grau de linearidade da isoterma.

Os valores $K_{f}$ e $n$ são estimados ajustando-se o modelo aos dados experimentais. A constante $\mathrm{K}_{\mathrm{f}}$ fornece uma medida da extensão da sorção e tem sido correlacionada com várias características dos solos. Na condição em que n é igual a 1, a sorção é diretamente proporcional à concentração da solução de equilíbrio e, neste caso, a constante $\mathrm{K}_{\mathrm{f}}$ é semelhante ao coeficiente de distribuição $\mathrm{K}_{\mathrm{d}}{ }^{26}$.

Dentre as características do solo, o teor de matéria orgânica é a que tem apresentado melhor correlação com a constante de Freundlich para a maioria dos herbicidas de amplo uso no Brasil. Todavia, outras caracteríscas como teor de argila e pH são também de grande importância ${ }^{27}$.

Desta forma, objetivou-se neste trabalho avaliar o comportamento da sorgoleona em um Latossolo Vermelho-Amarelo, típico do cerrado brasileiro, onde o sorgo é amplamente cultivado para produção de grãos, forragem e fornecimento de palhada para implantação do sistema de plantio direto. 


\section{PARTE EXPERIMENTAL}

\section{Procedimentos gerais}

Amostras de um Latossolo Vermelho-Amarelo típico da região do cerrado brasileiro foram coletadas na profundidade de 0 a 20 $\mathrm{cm}$, no município de João Pinheiro - MG. Essas amostras foram secas à temperatura ambiente, desagregadas e passadas por peneira de $2 \mathrm{~mm}$ sendo submetidas, em seguida, a análises físicas e químicas, de acordo com metodologia descrita na literatura ${ }^{28}$.

A sorgoleona foi obtida a partir de raízes de plantas de sorgo, conforme procedimento descrito por Ferreira e colaboradores ${ }^{29}$. A purificação desse composto foi realizada por cromatografia líquida de alta eficiência (CLAE) em um aparelho Shimadzu, CBM10A, equipado com um detector UV-VIS SPD-10AV, operando em comprimento de onda $(\lambda)$ de $280 \mathrm{~nm}$, uma bomba LC-6AD e um injetor Rheodyne com "loop" de $1 \mathrm{~mL}$. Utilizou-se como fase estacionária uma coluna semi-preparativa de fase reversa Shim-pack PREP-ODS C-18 (250 x 20 mm, 15 um e diâmetro dos poros de 100 Å) e, como fase móvel, uma mistura constituída de acetonitrila: água:ácido acético glacial (75:22:3), com fluxo de $6 \mathrm{~mL} \mathrm{~min}^{-1}$, sendo o tempo total de análise de $200 \mathrm{~min}$.

Para cada purificação foram utilizadas $0,10 \mathrm{~g}$ de amostra, sendo recolhidas 50 frações com volume de $15 \mathrm{~mL}$ cada. Essas frações foram posteriormente analisadas por CLAE, utilizando-se uma coluna analítica de fase reversa Lichrosorb RP-18 (250 x 4 mm, $5 \mu \mathrm{m})$.

O sistema de solventes utilizado consistiu em uma mistura de acetonitrila:água:ácido acético glacial (75:22:3), com fluxo da fase móvel de $2 \mathrm{~mL} \min ^{-1} \mathrm{e}$ "loop" de $20 \mu \mathrm{L}$.

As frações semelhantes foram reunidas e concentradas em evaporador rotativo a $35^{\circ} \mathrm{C}$, sendo, posteriormente, mantidas sob pressão reduzida durante $24 \mathrm{~h}$ para a completa eliminação do ácido acético.

A quantificação da sorgoleona nos diversos experimentos foi relalizada por CLAE, nas condições experimentais descritas anteriormente. Para isso foi preparada uma curva de calibração utilizando o padrão de sorgoleona nas concentrações de 0,$2 ; 0,5 ; 1,0$; 2,$5 ; 5,0 ; 10,0 ; 20,0 ; 30,0 ; 40,0 ; 50,0 ; 60,0 ; 75,0 ; 80,0$ e $100 \mu \mathrm{gL}^{-1}$ em acetonitrila.

\section{Determinação do tempo de equilíbrio para a sorção da sorgoleona}

A uma amostra contendo $1,0 \mathrm{~g}$ de solo em um erlenmeyer, foram adicionados $9,9 \mathrm{~mL}$ de solução de $\mathrm{CaCl}_{2} 0,01 \mathrm{~mol} \mathrm{~L}^{-1}$ e $0,1 \mathrm{~mL}$ de solução-padrão de sorgoleona, para que fosse obtida uma concentração final do padrão de $50 \mu \mathrm{g} \mathrm{mL} \mathrm{m}^{-1}$, em cada experimento. $\mathrm{O} \mathrm{pH}$ do meio foi ajustado para 5,30 $\pm 0,05$ e a mistura foi colocada sob agitação horizontal à temperatura de $25 \pm 1{ }^{\circ} \mathrm{C}$. Os tempos de equilíbrio avaliados foram 0,$25 ; 6 ; 12 ; 24 ; 36 ; 48$ e 72 h. Os sobrenadantes foram centrifugados a $1.108 \mathrm{~g}(3000 \mathrm{rpm})$ por $10 \mathrm{~min}$ e transferidos para frascos de vidro de $10 \mathrm{~mL}$. Este procedimento foi realizado em triplicatas, sendo que para cada repetição foram utilizados dois controles, dos quais um continha o aleloquímico sem o solo e o outro, o solo sem o aleloquímico.

Para a escolha da faixa de concentração da sorgoleona no estudo da sorção utilizaram-se $9,9 \mathrm{~mL}$ de solução de $\mathrm{CaCl}_{2} 0,01 \mathrm{~mol} \mathrm{~L}^{-1}$ e $0,1 \mathrm{~mL}$ de solução-padrão de sorgoleona, para que fossem obtidas concentrações finais do padrão de $10 ; 20 ; 30 ; 40 ; 50 ; 100 ; 150$; $200 \mu \mathrm{g} \mathrm{mL}^{-1} \mathrm{e}$, posteriormente, nas concentrações de 50; 100; 150; 200; 300; 400; 500; 700 e $1000 \mu \mathrm{g} \mathrm{mL} \mathrm{m}^{-1}$. As soluções foram mantidas sob agitação magnética por $24 \mathrm{~h}$. Os tratamentos analisados foram realizados com quatro repetições e um controle, constituído pelo aleloquímico sem o solo.
A isoterma de sorção foi obtida utilizando-se soluções de sorgoleona, nas concentrações de 20; 30; 40; 50; 100; 150; 200 e $300 \mu \mathrm{g} \mathrm{mL}^{-1} \mathrm{em} \mathrm{CaCl}_{2} 0,01 \mathrm{~mol} \mathrm{~L}^{-1}$, com tempo de agitação de $24 \mathrm{~h}$ e uma massa de solo de 1,0 g para um volume total de $10 \mathrm{~mL}$.

Para o cálculo da quantidade de sorgoleona sorvida no solo utilizou-se a equação: $\mathrm{X} / \mathrm{m}=\left(\mathrm{C}_{\mathrm{p}}-\mathrm{C}_{\mathrm{e}}\right) \mathrm{v} / \mathrm{m}$, onde $\mathrm{X} / \mathrm{m}$ é a quantidade de sorgoleona sorvida por grama de solo $\left(\mu \mathrm{g} \mathrm{g}^{-1}\right)$; $\mathrm{v}$, o volume da solução no meio reacional $(10 \mathrm{~mL}) ; \mathrm{m}$, a massa de solo $(\mathrm{g}) ; \mathrm{C}_{\mathrm{p}}$, a concentração inicial de sorgoleona $\left(\mu \mathrm{gL}^{-1}\right)$ e $\mathrm{C}_{\mathrm{e}}$, a concentração de sorgoleona em equilíbrio com o solo $\left(\mu \mathrm{g} \mathrm{mL}^{-1}\right)$.

\section{Estudo da persistência e da degradação da sorgoleona no solo}

A persistência e degradação da sorgoleona no solo foram avaliadas adicionando-se duas doses do aleloquímico em erlenmeyers de $125 \mathrm{~mL}$ contendo $10 \mathrm{~g}$ de solo, de modo a se obter concentrações finais de sorgoleona de 300 e $1000 \mu \mathrm{g} \mathrm{g}^{-1}$. Os erlenmeyers foram envolvidos em folhas de alumínio e acondicionados ao acaso em germinador mantido à temperatura de $27{ }^{\circ} \mathrm{C}$ e umidade de $100 \%$. O experimento foi avaliado após o período de 7, 14, 21, 28, 60 e 90 dias. Para cada dose foram feitas quatro repetições e um controle, contendo o aleloquímico sem o solo. A sorgoleona do solo foi posteriormente extraída utilizando-se diclorometano (4 x $5 \mathrm{~mL}$ ) com agitação horizontal por $30 \mathrm{~min}$. Após cada extração, a concentração de sorgoleona foi determinada por CLAE, utilizando-se as condições experimentais previamente descritas.

O tempo necessário para a extração da sorgoleona do solo foi determinado adicionando-se, em erlenmeyers de $250 \mathrm{~mL}$, amostras de $10 \mathrm{~g}$ de solo, fortificadas com padrão de sorgoleona, de modo a se obter a concentração de $50 \mu \mathrm{g} \mathrm{g}^{-1}$. As misturas foram deixadas em repouso por $24 \mathrm{~h}$ e, após esse tempo, as amostras foram submetidas a três extrações sucessivas com $20 \mathrm{~mL}$ de diclorometano, sob agitação horizontal por 5, 10, 15, 20 e $30 \mathrm{~min}$. O sobrenadante foi filtrado e transferido quantitativamente para balões de fundo redondo. Após redução do volume em evaporador rotativo, o resíduo foi transferido para um balão volumétrico de $10 \mathrm{~mL}$, aferido com diclorometano e analisado por CLAE.

\section{RESULTADOS E DISCUSSÃO}

A amostra de solo utilizada apresentou as seguintes características físicas e químicas: teor de argila $28 \%$, silte $7 \%$, areia $29 \%$,

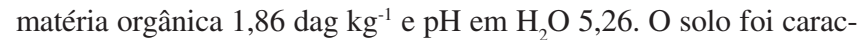
terizado como de textura franco argilo-arenoso, sendo considerado como representativo dos solos sob cerrado, onde o sorgo é amplamente cultivado.

Devido à não disponibilidade comercial da sorgoleona, a mesma foi obtida a partir de exsudatos de raízes de sorgo, utilizandose o procedimento previamente descrito na literatura ${ }^{29}$. Esse esxudato $(100 \mathrm{mg})$ foi submetido àa análise por cromatografia líquida de alta eficiência semi-preparativa, o que resultou na obtenção de aproximadamente $80 \mathrm{mg}$ do composto desejado. A pureza do composto obtido foi avaliada por CLAE, e o cromatograma resultante, mostrado na Figura 2, apresentou apenas um pico com tempo de retenção de aproximadamente 4,7 min. A sorgoleana apresentou-se como um sólido alaranjado com temperatura de fusão igual a $46,2-48,5^{\circ} \mathrm{C}$. A análise do espectro no infravermelho desse composto revelou a presença de uma banda em $3340 \mathrm{~cm}^{-1}$, indicando a existência de grupo hidroxila na molécula. A banda em 1637 $\mathrm{cm}^{-1}$ é devida ao estiramento da ligação $\mathrm{C}=\mathrm{O}$, conjugada a uma ligação dupla, conforme esperado para quinonas. A absorção em $1596 \mathrm{~cm}^{-1}$ resulta do estiramento da ligação $\mathrm{C}=\mathrm{C}$. Os dados de RMN de ${ }^{1} \mathrm{H}$ e de ${ }^{13} \mathrm{C}$, obtidos para o composto isolado, mostraram-se 


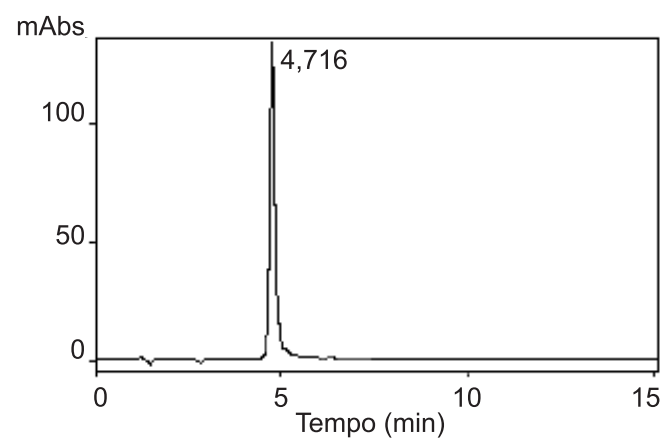

Figura 2. Cromatograma do padrão da sorgoleona na concentração de $75 \mu \mathrm{g} m L^{-1}$

idênticos aos publicados para a sorgoleona ${ }^{29}$. Esse composto foi então utilizado nos diversos experimentos.

A quantificação da sorgoleona em todos os experimentos foi realizada por meio da curva de calibração preparada com padrão de sorgoleona nas concentrações de 0,$2 ; 0,5 ; 1,0 ; 2,5 ; 5,0 ; 10,0$; 20,0; 30,0; 40,0; 50,0; 60,0; 75,0; 80,0 e $100 \mu \mathrm{g} \mathrm{mL} \mathrm{m}^{-1} \mathrm{em}$ acetonitrila, onde se obteve a equação: Absorvância $=21318$ x concentração de sorgoleona $-4053,7$, com $r^{2}=0,9997$.

O resultado do experimento sobre o tempo de equilíbrio para a adsorção da sorgoleona é mostrado na Figura 3, onde se verifica que um tempo mínimo de $24 \mathrm{~h}$ foi requerido para que o equilíbrio se estabelecesse. No estudo da interação fase sólida-solução, são vários os fatores que alteram o tempo de equilíbrio de sorção de uma espécie em solução por um solo. De modo geral, o tempo de equilíbrio é dependente da estrutura do composto e das características do solo.

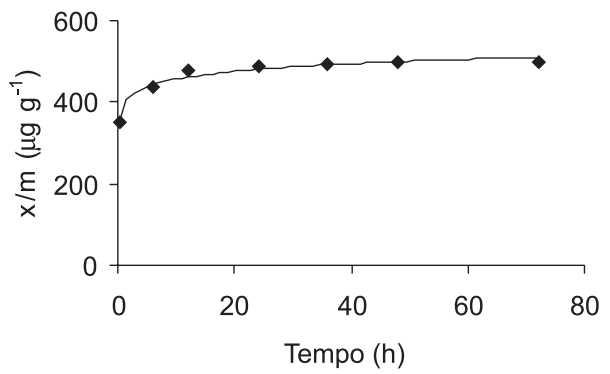

Figura 3. Efeito do tempo sobre a sorção da sorgoleona na amostra do solo estudada

No experimento desenvolvido para quantificar a sorgoleona no solo, utilizaram-se dois tipos de controle. Em um deles foi utilizado o solo sem a sorgoleona e no outro, a sorgoleona sem o solo. A função do primeiro foi observar a ocorrência de picos interferentes no cromatograma provenientes da matéria orgânica do solo, o que não foi constatado. A função do segundo foi observar a possível diminuição na concentração do aleloquímico devido à degradação durante o experimento, no entanto, esta possibilidade foi descartada.

Dentro de certos valores de concentração, a equação de Freundlich descreve de maneira adequada a sorção, apresentando limitações para concentrações muito elevadas ${ }^{30}$. Sendo assim, tornou-se necessário determinar a faixa adequada de concentração da sorgoleona, para construir as isotermas de Freundlich. Como a literatura não apresenta nenhum trabalho dessa natureza com a sorgoleona, foram desenvolvidos dois experimentos, sendo um deles constituído por soluções de concentrações finais variando de 10 a $200 \mu \mathrm{g} \mathrm{mL}^{-1}$, e o outro por soluções de concentrações finais variando de 50 a $1000 \mu \mathrm{g} \mathrm{mL} \mathrm{m}^{-1}$.
Nas Figuras 4a e 4b verifica-se a relação da quantidade de sorgoleona sorvida $(\mathrm{x} / \mathrm{m})$ em função das concentrações de equilíbrio (Ce). Observa-se (Figura 4b) que até a concentração sorvida de aproximadamente $3.000 \mu \mathrm{g} \mathrm{g} \mathrm{g}^{-1}$, que corresponde à concentração de

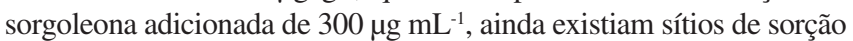
capazes de interagir com a sorgoleona. Acima desta concentração ocorreu saturação de tais sítios, pois praticamente toda a sorgoleona adicionada permaneceu no sobrenadante sem ser sorvida.
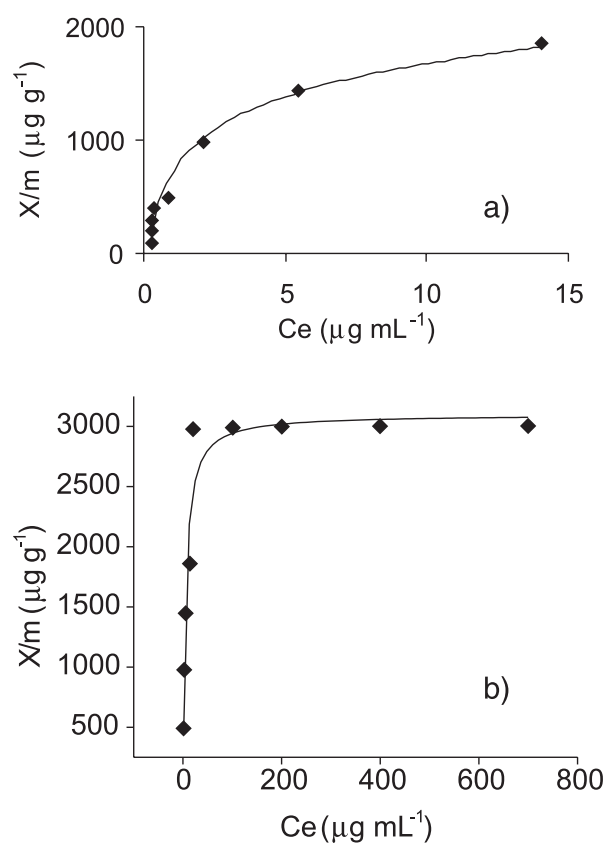

Figura 4. Quantidade de sogoleona sorvida $(x / m)$ em função de suas concentrações no equilíbrio

Com base nesses resultados utilizaram-se as concentrações iniciais de 20, 30, 40, 50, 100, 150, 200 e $300 \mu \mathrm{g} \mathrm{mL} \mathrm{m}^{-1}$ de sorgoleona para a obtenção da isoterma de Freundlich $\left(\mathrm{x} / \mathrm{m}=538,62 \mathrm{Ce}^{0,5481}\right.$, $\mathrm{R}^{2}=0,9655$ ) (Figura 5), a qual permitiu descrever a relação entre as concentrações da sorgoleona, em solução e em fase sólida, e obter os valores de $\mathrm{K}_{\mathrm{f}}$ e $\mathrm{n}$.

A isoterma de sorção encontrada enquadra-se no tipo-L, de acordo com a classificação proposta por Giles $^{31}$, indicando uma alta afinidade do solo em relação à sorgoleona.

Verificou-se que a equação de Freundlich ajustou-se adequadamente para descrever a sorção da sorgoleona no substrato utilizado, uma vez que o coeficiente de determinação encontrado foi elevado $\left(\mathrm{R}^{2}=0,9655\right)$. $\mathrm{O}$ alto valor do coeficiente de Freundlich encontrado $\left(K_{f}=538,62\right)$ indica que a capacidade de sorção da matriz de solo é muito elevada.

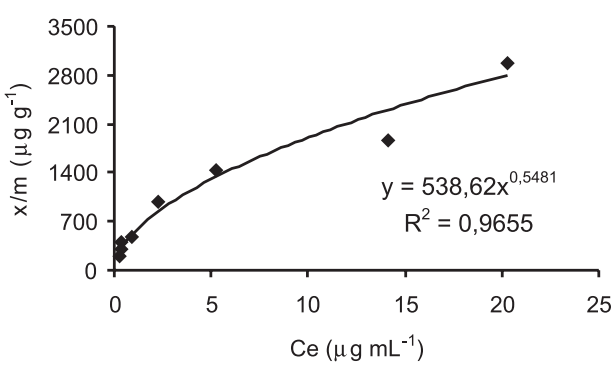

Figura 5. Isoterma de Freundlich da sorgoleona na amostra de solo estudada 
Este resultado evidenciou a necessidade de se utilizar concentração elevada de sorgoleona no solo para a realização do experimento de degradação. Caso contrário, todo o composto seria sorvido pelo solo. Além disso, antes de se iniciar o estudo da persistência da sorgoleona no solo fez-se um trabalho preliminar para determinar o melhor tempo de agitação para extração deste composto a partir do solo. Para isto, foram testados os tempos de 5, 10, 15, 20 e $30 \mathrm{~min}$. Os resultados obtidos indicaram que o tempo de contato de 15 min de agitação da mistura entre o solvente extrator e a amostra de solo foi suficiente para a recuperação de $89 \%$ da sorgoleona. No entanto, a melhor recuperação (93\%) foi conseguida com 30 min de contato (Figura 6).

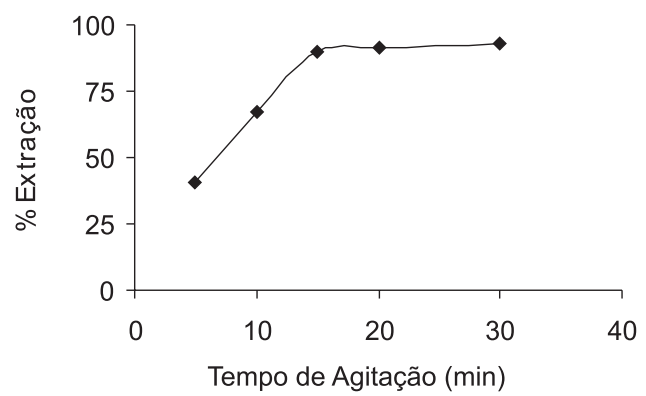

Figura 6. Porcentagem de recuperação da sorgoleona da matriz de solo em função do tempo de extração, utilizando-se DCM como solvente extrator

A persistência da sorgoleona no solo foi avaliada através das medidas dos teores recuperados a partir da matriz de solo, ao longo de 90 dias. No primeiro mês, os teores de sorgoleona foram analisados a cada 7 dias, e nos dois meses seguintes, a cada 30 dias (Figura 7).

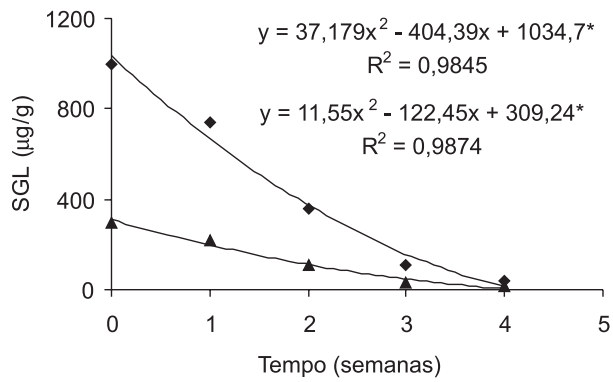

Figura 7. Teores de sorgoleona recuperados do solo em função do tempo após a aplicação, nas concentrações iniciais de $1000 \mu \mathrm{g} \mathrm{mL^{-1 }}$ ( ) $e$ $300 \mu \mathrm{mg} \mathrm{mL}^{-1}(\mathbf{\Delta})$

A persistência de um composto pode ser medida pelo tempo de meia-vida $\left(\mathrm{t}_{1 / 2}\right)$, que é o período de tempo necessário para que a concentração inicial do composto seja reduzida à metade. No caso da sorgoleona o tempo de meia-vida foi de 10 dias nas condições estudadas. Um herbicida é considerado não persistente quando seu tempo de meia-vida é inferior a 30 dias, embora alguns considerem levemente persistentes herbicidas que têm tempo de meia-vida entre 5 e 21 dias $^{32}$.

Após a análise de regressão, observou-se que o modelo polinomial quadrático foi o que melhor descreveu o processo de degradação da sorgoleona no solo. As equações quadráticas obtidas $\left(\mathrm{SGL}_{(1000 \mu \mathrm{g} / \mathrm{g})}=37,179 \mathrm{t}^{2}-404,39 \mathrm{t}+1034,7 \mathrm{R}^{2}=09845 \mathrm{e}\right.$ $\left.\mathrm{SGL}_{(300 \mu \mathrm{g} / \mathrm{g})}=11,55 \mathrm{t}^{2}-122,45 \mathrm{t}+309,24 \mathrm{R}^{2}=0,9874\right)$ nos permitem quantificar a rápida degradação da sorgoleona no solo, não sendo mais detectado qualquer resíduo 60 dias após a aplicação.
Os resultados obtidos no presente trabalho concordam com os relatados por Czarnota e colaboradores ${ }^{13}$. No entanto, Einhellig e Souza $^{11}$ relataram que houve recuperação de sorgoleona no solo um ano após o cultivo de sorgo, o que pode estar relacionado com as características do solo utilizado.

A rápida redução no teor de sorgoleona nas condições estudadas talvez se deva, em parte, àa alta umidade na qual ela foi exposta ou pelo baixo teor de matéria orgânica da amostra de solo, tendo em vista que a persistência de alguns compostos é muito reduzida em condições de alta umidade como, por exemplo, algumas trizinas $^{33}$.

\section{CONCLUSÃO}

Pelos dados obtidos conclui-se que a isoterma de Freundlich é adequada para descrever a sorção da sorgoleona no solo, a julgar pelo elevado coeficente de correlação encontrado $\left(R^{2}=0,9655\right)$, enquadrando-se no tipo-L, o que indica alta afinidade dos sítios de sorção pela sorgoleona. Os coeficientes de Freundlich $\left(\mathrm{K}_{\mathrm{f}}=538,62 \mathrm{e}\right.$ $1 / \mathrm{n}=0,5481$ ) revelaram alta capacidade de sorção e restrita mobilidade da sorgoleona no solo. O tempo de meia-vida da sorgoleona no solo foi avaliado em 10 dias, nas condições estudadas, o que a classifica como não persistente ${ }^{18}$. $\mathrm{O}$ modelo polinomial quadrático foi o que melhor descreveu a degradação da sorgoleona no solo. Após 60 dias de incubação não foi mais detectado qualquer resíduo de sorgoleona no solo. Assim sendo, caso algum efeito fitotóxico ocorra após este período, provavelmente não será devido à sorgoleona, mas possivelmente devido aos produtos de degradação da mesma.

\section{AGRADECIMENTOS}

Ao Conselho Nacional de Desenvolvimento Cientifico e Tecnológico (CNPq) pela bolsa de estudo (L. S. Chinelatto Jr.) e pelas bolsas pesquisa (A. J. Demuner, A. A. Silva e L. C. A. Barbosa) e à Fundação de Pesquisa de Minas Gerais (FAPEMIG) pelo apoio financeiro. Os autores também agradecem ao estudante R. C. Werlang, pela contribuição durante a realização deste trabalho.

\section{REFERÊNCIAS}

1. Reigosa, M. J.; Pedrol, N.; Allelopathy: From molecules to ecosystems, Science Publishers Inc.: Plymouth, 2002.

2. Einhellig, F. A. Em Allelopathy: Organisms, processes and aplications; Inderjit, D.; Einhellig, F. A., eds.; Am. Chem. Soc., 1995, p. 1-24.

3. Panasiuk, O.; Bills, D. D.; Leather, G. R.; J. Chem. Ecol. 1986, 12, 1533.

4. Einhellig, F. A.; Rasmunssen, J. A.; J. Chem. Ecol. 1989, 115, 951.

5. Martin, J. H.; Couch, J. F.; Briese, R. R.; J. Am. Soc. Agron. 1938, 30, 725.

6. Guenzi, W. D.; McCalla, T. M.; Agron. J. 1966, 58, 303.

7. Guenzi, W. D.; McCalla, T. M.;Nordstadt, F. A.; Agron. J. 1967, 59, 163.

8. Stafford, H. A.; Plant Physiol. 1968, 43, 318.

9. Netzly, D. H.; Butler, L. G.; Crop Sci. 1986, 26, 775.

10. Netzly, D. H.: Riopel, J. L.; Ejeta, G.; Butler, L. G.; Weed Sci. 1988, 36, 441

11. Einhellig, F. A.; Souza, I. F.; J. Chem. Ecol. 1992, 18, 11.

12. Bem-Hammouda, M.; Kremer, R. J.; Minor, H. C.; Crop Sci. 1995, 35 1652 .

13. Czarnota, M. A.; Paul, R. N.; Dayan, F. E.; Chandrashekhar, I. N.; Weston, L. A.; Weed Tecnol. 2001, 15, 813.

14. Rasmussen, J. A.; Helj, A. M.; Einhelig, F. A.; Thomas, J. A.; J. Chem. Ecol. 1992, 18, 197.

15. Rimando, A. M.; Dayan, F. E.; Czarnota, M. A.; Weston, L. A.; Duke, S. O.; J. Nat. Prod. 1998, 61, 927.

16. Barbosa, L. C. A.; Ferreira, M. L.; Demuner, A. J.; Silva, A. A.; Pereira, R. C.; Quim. Nova 2001, 24, 751 .

17. Lima, L. S.; Barbosa, L. C. A.; Alvarenga, E. S.; Demuner, A. J.; Silva, A. A.; Aust. J. Chem. 2003, 56, 625.

18. Reis, C.; Fabris, J. D.; Novais, R. F.; Bahia Filho, A. F. C.; Santana, D. P.; Curi, N.; Coey, J. M. D.; Rev. Bras. Ci. Solo 1995, 19, 337. 
19. Graham-Bryce, I. J.; J. Sci. Food Agric. 1967, 18, 72.

20. Lanças, F. M.; Vilegas, J. H. Y.; Galhiane, M. S.; Pesticidas Revista Técnico Científica 1994, 4, 39.

21. Tavares, M. C. H.; Landgraf, M. D.; Vieira, E. M.; Rezende, M. O. O.; Quim. Nova 1996, 19, 605.

22. Kuo, W. S.; Regan, R. W.; J. Environ. Sci. Health 1999, 34, 431

23. Vieira, E. M.; Prado, A. G. S.; Landgraf, M. D.; Rezende, M. O. O.; Quim Nova 1999, 22, 305.

24. Lopes, N. P.; Queiroz, M. E. L. R.; Neves, A. A.; Quim. Nova 2002, 25, 544.

25. Gunary, D. A.; Soil Sci. 1970, 21, 72.

26. Steveson, F. J.; Humus Chemistry: Genesis, Composition, Reactions, John Wiley \& Sons: New York, 1994.
27. Francioso, O.; Bak, E.; Rossin, N.; Saqui, P.; Sci.Total Environ. 1992, 123/ 124, 503.

28. EMBRAPA; Manual de Métodos de Análise de Solo, Sociedade Brasileira de Ciência do Solo: Rio de Janeiro, 1997.

29. Ferreira, M. L.; Barbosa, L. C. A.; Demuner, A. J.; Silva, A. A.; Wakil, J.; Acta Scientiarum 1999, 21, 565

30. Barrow, N. J.; J. Soil Sci. 1978, 29, 447.

31. Giles, C. H.; McEvans, T. H.; Nakhwa, S. N.; J. Chem. Soc. 1960, 3, 3973.

32. Silva, A. A.; Silva, J. F.; Ferreira, F. A.; Ferreira, L. R.; Silva, J. F.; Curso de proteção de plantas, módulo 3, Abeas: Brasilia, 1999.

33. Moyer, J, R.; Blackshaw, R. E.; Weed Technol. 1993, 7, 988. 